\title{
Revisiones
}

\section{Teletrabajo, un enfoque desde la perspectiva de la salud laboral}

\section{Teleworking, an approach from the perspective of occupational health}

\author{
Nelson W. López 1,4, M. Claudia Pérez-Simon 2,4, Edwige G. Nagham-Ngwessitcheu 3,4, María Vázquez-Ubago 3,4 \\ 1. Hospital Universitario Basurto, Bilbao. España. \\ 2. Hospital Universitario Txagorritxu, Vitoria. España. \\ 3. Hospital Universitario Cruces, Barakaldo. España. \\ 4. Unidad Docente de Medicina del Trabajo del País Vasco. España.
}

Recibido: 23-01-14

Aceptado: 17-07-14

\section{Correspondencia}

Nelson Walter López.

Servicio de Prevención de Riesgos Laborales.

Hospital Universitario Basurto.

Avenida Montevideo n. ${ }^{\circ} 18$.

48013. Bilbao. España

Teléfono 944006239

Correo electrónico: nelsonwalter.lopez@gmail.com

\section{Resumen}

El desarrollo de las TIC favoreció la aparición del Teletrabajo (TT) a mediados del siglo pasado, pero actualmente, tan sólo el 13,2\% de las empresas españolas lo fomentan. Las diferencias culturales, sociales, y las dificultades para homogeneizar la información disponible, hacen que resulte un desafío unificar criterios para su desarrollo.

Esta revisión bibliográfica pretende mostrar el marco global del TT en España, factores de riesgo, repercusión, beneficios y perjuicios, detallar las condiciones y medidas de seguridad laboral que deberían acompañar el proceso. Para obtener los artículos se ha realizado una búsqueda bibliográfica en las principales bases de datos biomédicas.

Efectos beneficiosos evidenciados serían el alto nivel de autonomía, elevada satisfacción laboral, mejor relación con el supervisor, disminución de la tasa de abandono del trabajo y del conflicto familiar. Otras publicaciones indican que reforzando los recursos personales, mejora el compromiso y la eficacia de los trabajadores.

Se destaca la escasa existencia de descriptores específicos DeCS-MeSH para TT, incluidos en las bases de datos bibliográficas que utilizan estos tesauros como motor de búsqueda, Medline, Cochrane Library e IBECS, lo que dificulta la recuperación de información.

El desconocimiento general sobre esta modalidad de trabajo, la cultura organizacional, el sistema de control y supervisión laborales basadas en la desconfianza, no facilitan la implantación del TT en España al ritmo en el que se impone en otros países de nuestro entorno como Reino Unido, Alemania y Francia, por lo que se hace necesario un cambio de mentalidad colectiva para integrar el TT como alternativa laboral en el marco de la sociedad actual en la que nos desenvolvemos.

Med Segur Trab (Internet) 2014; 60 (236) 587-599

Palabras clave: Teletrabajo, TT, Tecnologías de información y comunicación, TIC. 


\section{Abstract}

The development of TIC contributed to the emergence of Telecommuting (TT) in the middle of the last century, but nowadays only the $13.2 \%$ of Spanish companies promote it. The cultural and social differences, added to the difficulties to standardize the available information, make it a challenge to unify criteria for its development.

The aim of this bibliographic review is to show the global framework of TT in Spain, the risk factors, impact, benefits and drawbacks, detailing the conditions and occupational safety measures that should accompany the process. In order to obtain the articles, a bibliographic research was realized in the principal biomedical databases.

It has been evidenced that some beneficial effects would be the high level of autonomy, higher labor satisfaction, better relationship with the supervisor, decreasing of work dropout rate and family conflict. Other publications indicate that strengthening the personal resources, improve the commitment and efficiency of workers.

It should be emphasized that the low existence of specific DeCs-MeSH descriptors for TT included in the bibliographical databases using these thesauros as search engine, Medline, Cochrane Library and IBECS, which makes very difficult the recovery of information.

The general lack of knowledge about this type of work, the organizational culture, the control and labour supervision system based on mistrust, do not facilitate the implementation of the TT in Spain at the same place as other neighboring countries such as UK, Germany and France impose, this is why it becomes necessary a change of collective mentality to integrate the TT as an alternative employment within the society in which we live.

Med Segur Trab (Internet) 2014; 60 (236) 587-599

Keywords: Teleworking, TT, INFORMATION technology and communications, TICs. 


\section{INTRODUCCIÓN}

Desde hace un par de décadas el rápido desarrollo tecnológico y las innovaciones en la actual sociedad de la información, están modificando la manera con la que hemos de desarrollar nuestra actividad laboral, siendo el sector de las telecomunicaciones decisivo, al permitir el acceso al puesto de trabajo, sin necesidad de la presencia física del trabajador y surgiendo así una nueva forma de interacción laboral que se conoce como "teletrabajo" (TT). Sin embargo dicha expresión queda incompleta, si la misma no se realiza utilizando las tecnologías de la información y de la comunicación (TIC), dato clave para considerar una actividad profesional como TT.

Es en 1973 cuando Jack Nilles de EEUU, acuñó el término TT, en su original Telecommuting y lo definió "como aquella forma de enviar el trabajo al trabajador, en lugar de enviar el trabajador al trabajo" ${ }^{1}$.

La Comisión Europea designa al TT como «aquellas actividades ejercidas lejos de la sede de la empresa, a través de la comunicación diferida o directa por medio de las nuevas tecnologías"; más precisa es la definición que nos da la OIT: «forma de trabajo efectuada en un lugar alejado de la oficina central o del centro de producción y que implica una nueva tecnología que permite la separación y facilita la comunicación ${ }^{2}$.

Una definición completa es la de Salazar del 2007 «forma flexible de organización del trabajo; que consiste en el desempeño de éste fuera del espacio habitual de trabajo, durante una parte importante de su horario laboral, pudiendo realizarse a tiempo parcial o completo. Engloba una amplia gama de actividades y requiere el uso frecuente de TIC para el contacto entre el trabajador y la empresa. Pudiendo ser realizado por cualquier persona independiente del género, edad y condición física ${ }^{3}$.

Otras autoridades a nivel estatal y europeo, han dejado patente su preocupación; el Instituto Nacional de Seguridad e Higiene en el Trabajo (INSHT) a través de la Nota Técnica de Prevención 412 (NTP 412) sobre los criterios de implantación del TT4 (1996), y la Agencia Europea para la Seguridad y Salud en el Trabajo (AESST) en su boletín informativo del 2001 los acuerdos entre los sectores de las telecomunicaciones y el comercio sobre las directrices para el TT. Además en su informe 2013-2020 hace clara referencia a aspectos como la globalización, las nuevas tecnologías, el aumento en la proporción a nivel laboral de mujeres, inmigrantes y discapacitados, entre otros 5 .

Actualmente casi un $90 \%$ de las empresas británicas incentiva el TT, Francia y Alemania un 30\%, y tan solo el $13 \%$ de las españolas lo hacen ${ }^{6}$.

Debido al progreso del TT, a las diferencias culturales y sociales que influyen en el enfoque del mismo y a las dificultades para homogeneizar la información disponible, se presenta un reto en la unificación de criterios para el desarrollo de esta actividad.

Con esta revisión se pretende profundizar en el conocimiento del TT, como posibilidad laboral que no distingue género, edad ni condición física, reflejando los aspectos positivos y negativos del mismo, los factores de riesgo a los que están expuestos estos trabajadores, para poder comparar lo nuevo con lo precedente, y poder concretar la información disponible en la actualidad.

\section{METODOLOGÍA}

Se realizó la búsqueda bibliográfica en las siguientes bases de datos: MEDLINE (vía PubMed), WOK, OSH UPDATE, CISDOC, LILACS, COCHRANE LIBRARY, IBECS, SCOPUS y en repositorios como: Scielo y IOS press. Además se completó la búsqueda en GOOGLE.

Los términos DeCS que se utilizaron en la búsqueda fueron: telecommunications, risk factor, communications media, working conditions y los términos libres aplicados: telework, teleworker, teleworking y telecommuting. 
Se definieron diferentes estrategias de búsqueda utilizando descriptores «MeSHDeCS" para la búsqueda en las bases de datos MEDLINE (PubMed), Cochrane Library e IBECS y términos libres para las demás bases de datos referenciadas anteriormente.

La búsqueda en Google se realizó mediante lenguaje natural utilizando el término "Teletrabajo» (Literatura gris no convencional).

\section{PARA LA SELECCIÓN DE ARTíCULOS SE TUVIERON EN CUENTA LOS SIGUIEN- TES CRITERIOS}

\section{Criterios de inclusión}

- Artículos que aborden el TT desde el punto de vista laboral.

- Artículos publicados desde el 1 de enero de 2006 hasta el 31 de diciembre de 2013.

— Estudios publicados en español, inglés y francés.

\section{Criterios de exclusión}

- Artículos que describan el TT como trabajo físico realizado a domicilio.

- Artículos que aborden al TT desde el punto de vista exclusivamente empresarial.

- Artículos divulgativos o que no adopten un método científico para el estudio.

A partir de la estrategia de búsqueda se obtuvo una colección de 104 artículos. Tras eliminar duplicados, se realizó una selección de pertinencia doble ciego, resolviendo las discrepancias mediante consenso entre los componentes del equipo, obteniéndose un índice de Kappa con $90 \%$ de concordancia. A la colección resultante se le aplicaron los criterios de inclusión y exclusión referidos anteriormente quedando finalmente seleccionados para el estudio un total de 15 artículos (Tabla I). 


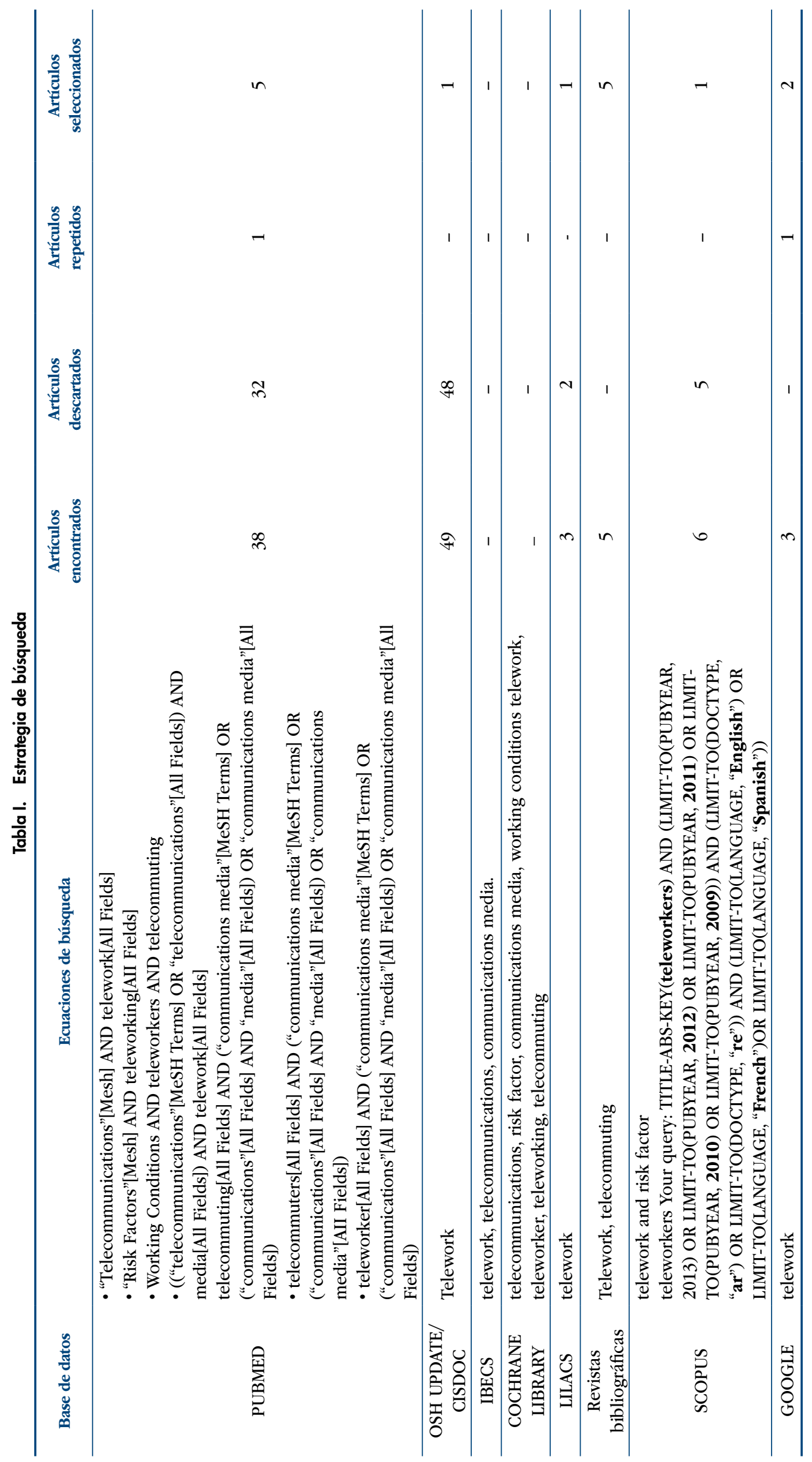

Teletrabajo, un enfoque desde la perspectiva de la salud laboral 


\section{Resultados}

Del periodo estudiado (2006-2013), el año 2012 es el que presenta mayor número de artículos publicados y el país que mayor producción científica genera en este campo de investigación es USA, pese a la gran variabilidad de enfoques y diseños con la que se aborda el tema del TT.

Entre los 15 artículos incluidos en el estudio, un metaanálisis menciona la autonomía, la relación familia-trabajo, satisfacción laboral y relación trabajador-supervisor como los principales beneficios que se pueden atribuir al TT. Incluso, aspectos positivos como: la disminución de intención de abandono o renuncia al puesto de trabajo y el estrés de origen laboral. La posibilidad de inclusión de discapacitados, personas mayores y mujeres con carga familiar también se destacó como otro aspecto positivo inherente al TT.

Igualmente en otras publicaciones, se alude al hecho de que se pueden potenciar los recursos individuales de los trabajadores y con esto impulsar la autoeficacia de los trabajadores (AET).

En países de Latinoamérica el desarrollo de las TIC es un medio propicio para atraer y retener profesionales cualificados.

Entre los efectos más negativos podemos destacar el aislamiento y la desmotivación como los principales perjuicios a los que el TT se expone.

En la Tabla II se presenta una síntesis de los principales resultados obtenidos en los diferentes trabajos analizados. 


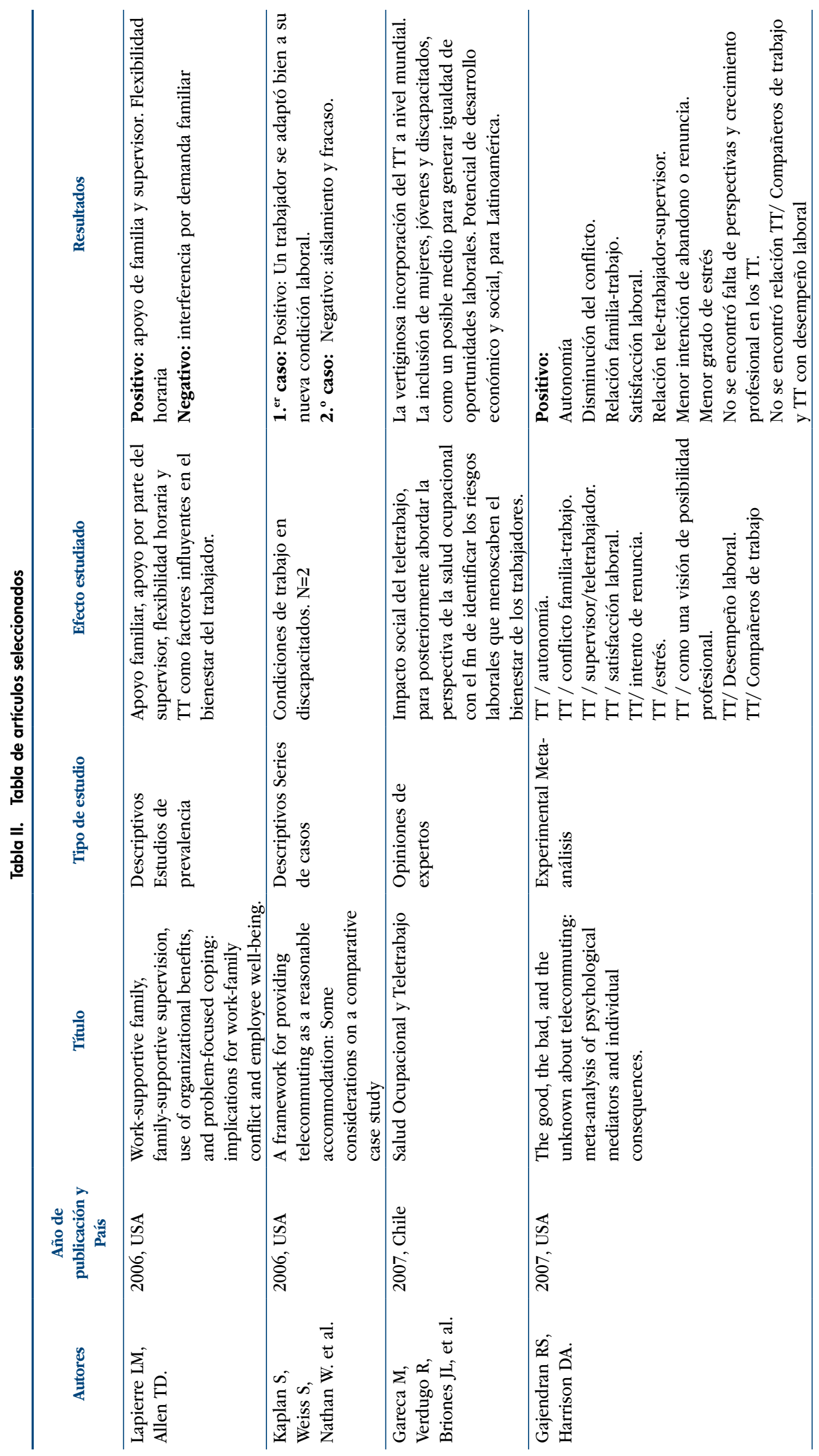




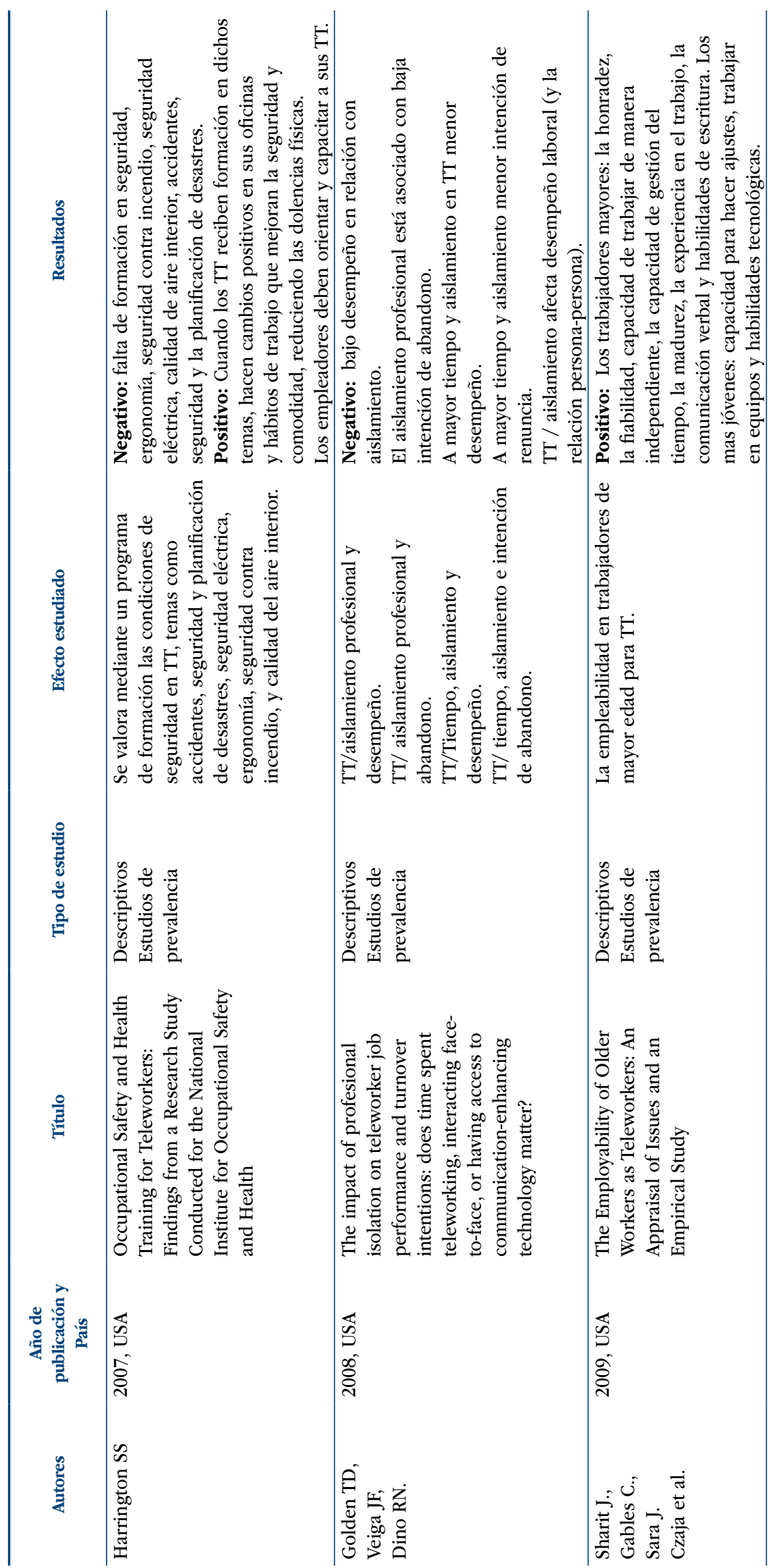

Teletrabajo, un enfoque desde la perspectiva de la salud laboral 


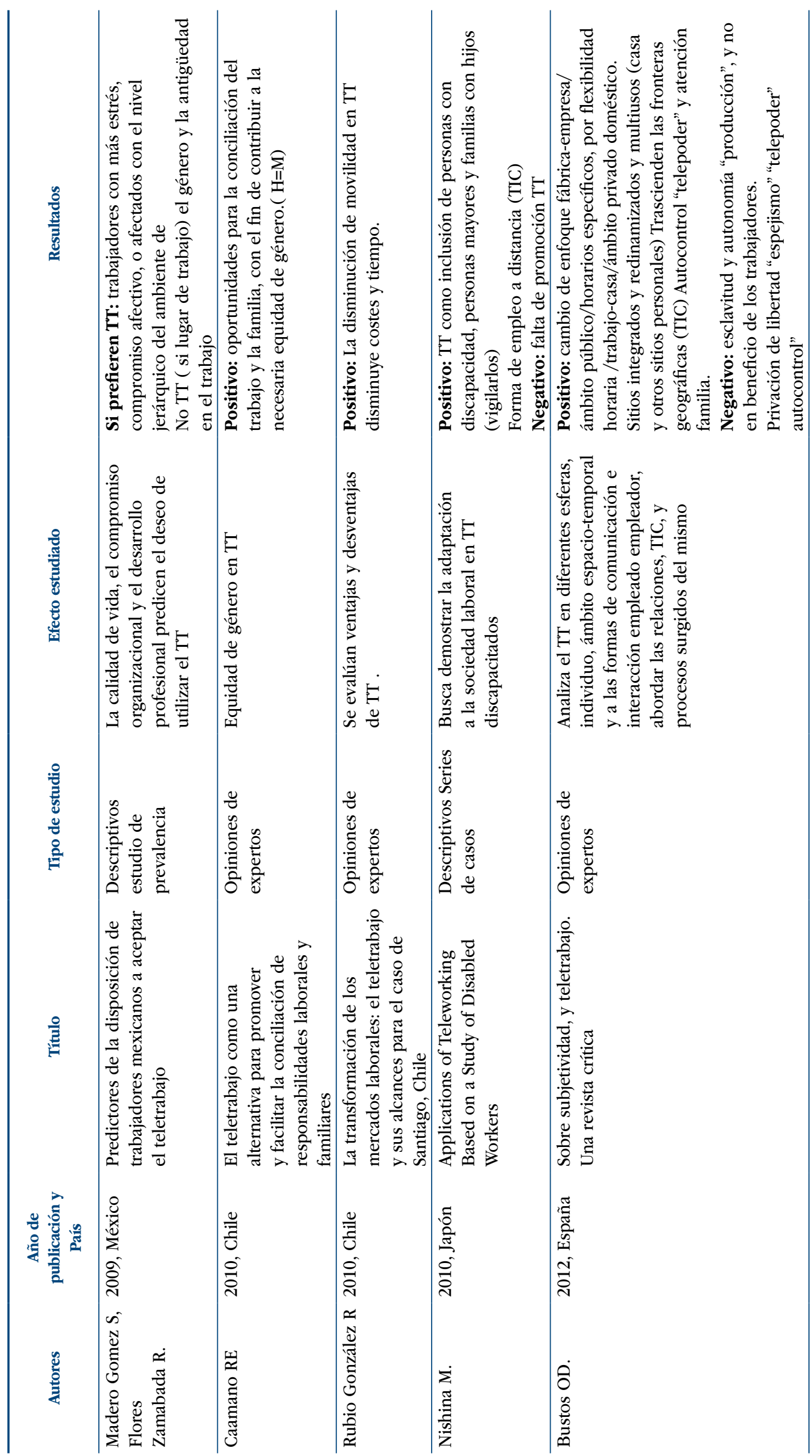




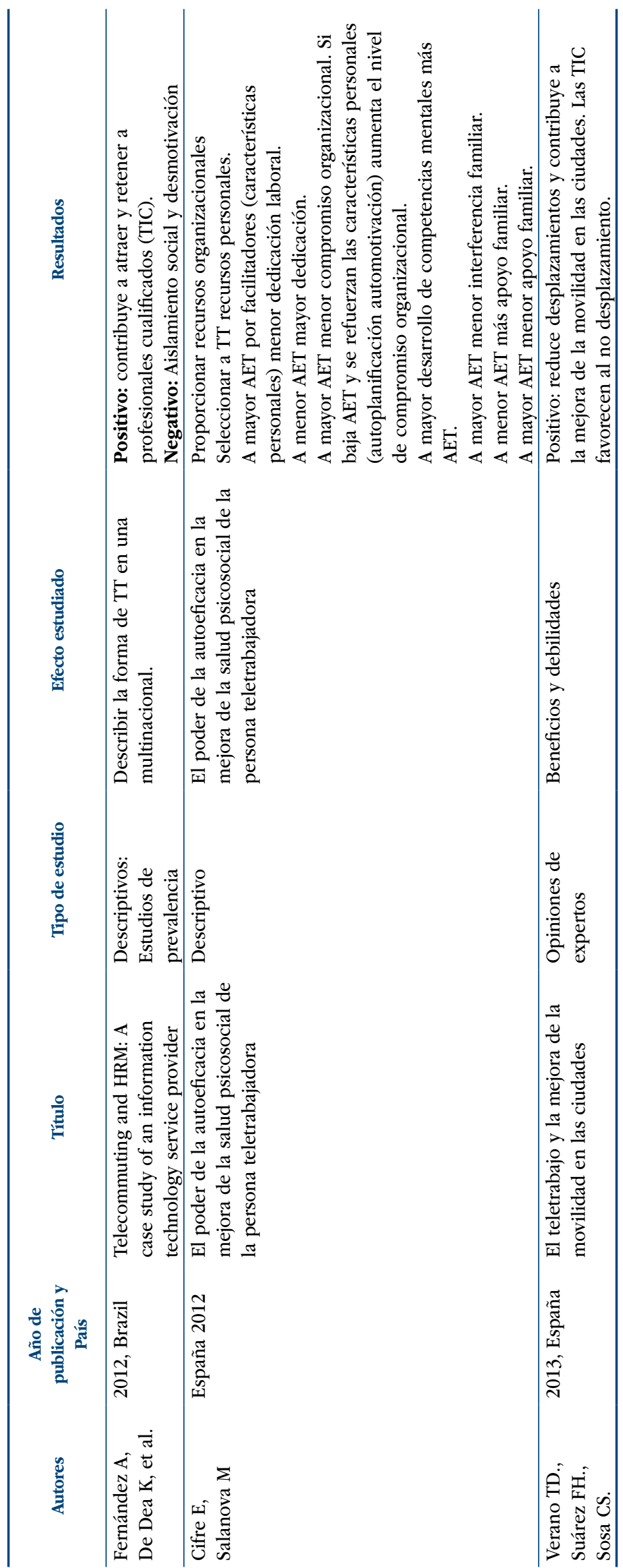




\section{DISCUSIÓN}

Aunque aún no ha pasado ni medio siglo desde el nacimiento del TT en Estados Unidos, su globalización e internalización en el sistema laboral, han supuesto grandes cambios y diferentes niveles de impacto, tanto para las empresas como para los trabajadores. Rubio R. sugiere, que el TT ejerce un impacto positivo sobre la organización espacial de los mercados laborales urbanos porque: disminuye la movilidad $^{7}$, favorece la flexibilidad horaria y trae como consecuencia para empleado y empleador una reducción de los costes en dietas y desplazamiento ${ }^{8}$.

En el lado opuesto de la balanza, Bustos $\mathrm{D}^{9}$ refiriéndose a los aspectos de productividad laboral relacionados con el TT, lo define: como una "esclavitud" porque el trabajador se encuentra sometido a una mayor carga horaria y laboral con el único fin de la rentabilidad empresarial, y por otro lado habla de "telepoder" como espejismo de una autonomía de la que realmente no dispone. En una dirección similar, Golden $\mathrm{T}^{10}$, relaciona las condiciones de aislamiento que rodean al TT con un menor desempeño laboral.

Entre los principales beneficios que esta modalidad laboral puede ofrecer al trabajador tendríamos que mencionar: el alto nivel de autonomía, el alcance de satisfacción laboral, una relación más beneficiosa con su supervisor, la disminución del abandono del trabajo y el menor nivel de conflicto familiar ${ }^{11}$.

También, Lapierre L. destaca como efecto positivo el apoyo familiar al trabajador en contraposición con la demanda de atención familiar que puede resultar perjudicial al interferir durante su jornada laboral ${ }^{12}$.

Cifre y Salanova (2006) reflejan que si se refuerzan los recursos personales, mediante técnicas como la autoplanificación, automotivación, etc., aumenta el nivel de compromiso organizacional y la eficacia de los trabajadores. También describen que cuanto más eficaz es el trabajador, gestiona mejor la interferencia familiar y mejora el desempeño del mismo ${ }^{13}$.

Por otra parte, Madero hace referencia a aquellos trabajadores que preferían el TT, y que resultaron ser los que sufrían mayor estrés laboral, los que se veían sometidos al efecto jerárquico de la empresa, o bien lo solicitaban por motivos de compromiso afectivo. Sin embargo observa, que aquellos trabajadores de mayor antigüedad en la empresa y los de género femenino, escogen permanecer en la institución de trabajo ${ }^{14}$.

Sharit J. estudió la empleabilidad y desempeño en trabajadores asociado a la edad, concluyendo que los trabajadores de mayor edad demostraban mayor honradez, fiabilidad, independencia y experiencia en su trabajo, mientras que los más jóvenes tenían más destreza para hacer ajustes y en el manejo de la tecnología ${ }^{15}$.

En este contexto, Fernandes establece el TT como una medida que contribuye a atraer profesionales cualificados, tomando en cuenta la innovación tecnológica que en países de economías emergentes como Brasil ha tenido un gran impulso ${ }^{16}$.Y otros autores, como una oportunidad laboral para mujeres, jóvenes y discapacitados (Gareca, 2007) ${ }^{17}$. Otros autores como Salazar C. y Baker P., coinciden también en describir el TT como una oportunidad para emplear a personas con discapacidad ${ }^{3,22-24}$.

Harrigton SS. en un artículo que estudia las condiciones laborales del TT, plantea que existe una clara necesidad de formación para el trabajador en temas como seguridad, ergonomía, planes contra incendio, calidad del aire interior, seguridad eléctrica, accidentes y planificación de desastres, al evidenciar que aún hay mucho por hacer en este campo en la actualidad ${ }^{18}$.

Caamaño E., hace alusión a la necesidad de promocionar la igualdad de género en los trabajadores que desempeñan este tipo de trabajo, sin olvidar los roles familiares ${ }^{19}$.

Robertson M., apoya dos de los factores positivos anteriormente descritos: la disminución del estrés desencadenado por la oficina y la mejor acomodación al puesto 
de trabajo de los discapacitados. Añade como nuevos aspectos favorables: la adaptación del individuo al ambiente de trabajo, la disminución de la frecuencia de enfermedad y del absentismo laboral, el aumento de la productividad y un mejor control del trabajo. Al hablar de aspectos perjudiciales destaca: el aislamiento, el alto nivel de carga laboral, el estancamiento profesional y el conflicto familiar ${ }^{20}$.

Estudios recientes hacen referencia a las condiciones del lugar de trabajo, señalando que el trabajador considera como ideal mantener en su domicilio las mismas condiciones de su puesto de trabajo de las que dispondría en la oficina. Por tanto, para garantizar que los trabajadores tengan un puesto de trabajo adecuado y seguro para sus necesidades, los empleadores deberían suministrar el mobiliario necesario o al menos contribuir económicamente a configurar el puesto de trabajo domiciliario.

En relación a la influencia de la familia y la efectividad de la oficina en casa como lugar de trabajo, poco se ha encontrado descrito ${ }^{21}$. El TT puede convertirse en un arma de doble filo para la conciliación de la vida personal y familiar con la vida laboral, ya que a veces gestionar al mismo tiempo las demandas familiares y las laborales puede causar problemas a los empleados/as, con las dificultades que ello conlleva para la conciliación ${ }^{25}$.

Entre las principales conclusiones cabría mencionar que el desconocimiento general sobre esta modalidad de trabajo, la cultura organizacional, el sistema de control y supervisión laborales basadas en la desconfianza, no facilitan la implantación del TT en España al ritmo en el que se impone en otros países de nuestro entorno como Reino Unido, Alemania y Francia, por lo que se hace necesario un cambio de mentalidad colectiva para integrar el TT como alternativa laboral en el marco de la sociedad actual en la que nos desenvolvemos.

Se ha visto que el hábito de acudir todos los días a la oficina, y cumplir con horario fijo y una rutina, es el ideal de muchos trabajadores. La flexibilidad necesaria para adaptarse a la globalización del mercado de trabajo está creciendo en la actualidad en lo que respecta al TT, sin embargo queda mucho por aportar en esta área. Es necesario una evolución cultural que permita al trabajador administrar correctamente su tiempo, cambiando la necesidad de cumplir un horario para alcanzar una meta.

También consideramos necesario profundizar en el desarrollo de un marco normativo apropiado, pues concentrar la dispersión del conocimiento del marco legal que recoge los protocolos a aplicar en estos trabajadores es aún una asignatura pendiente, ya que entendemos el TT como una forma de desarrollo laboral que no debe diferir de otras modalidades y al que se han de aplicar protocolos similares a los establecidos para otros trabajadores, adaptados según cada caso concreto. Los teletrabajadores son una población susceptible a riesgos laborales para los cuales no existe una evaluación planificada de los mismos. Entendemos de primordial importancia que se debe proporcionar a este tipo de trabajadores un puesto de trabajo libre de riesgo o peligro y establecer medidas de prevención equiparables a otros puestos de trabajo. En cuanto a las condiciones de seguridad en TT, poco hemos podido encontrar y sólo dos artículos publicados en lo últimos años hacían alguna mención a ello.

En España, solo una minoría de empresas y de población trabajadora contempla el TT como una alternativa laboral lo que concuerda con la necesidad de avanzar conceptualmente hacia un modelo laboral más integrado en la sociedad digital en la que nos movemos y profundizar en su conocimiento, pues cabe destacar el escaso número de artículos encontrados en estos 7 últimos años bajo los criterios de búsqueda utilizados en nuestra revisión, lo que puede ser debido a la metodología utilizada, a una escasez de producción científica abordando el tema o a la dificultad de encontrar descriptores específicos que faciliten la recuperación de la información.

Finamente podemos concluir que al tratarse de un tema laboral emergente y en reciente auge, la metodología utilizada por los diferentes autores y la variabilidad del enfoque hacia el abordaje del tema, representa una dificultad añadida a la hora de interpretar los resultados intentar unificar criterios para el análisis. 


\section{.}

\section{REFERENCIAS BIBLIOGRÁFICAS}

1. Nilles J. The telecomunication-transportation trade off. Options for tomorrow and today. Jala International. 1973:3.

2. Thibault J. El teletrabajo. CES. 2000:24.

3. Salazar C, Baker P. El Teletrabajo como aporte a la inserción laboral de personas con discapacidad en Chile: Una gran carretera virtual por recorrer. Ciencia y Trabajo. 2007; 9(24):89-98.

4. Pérez J, Sancho T, Nogareda C. NTP 412: Teletrabajo: Criterios para su implantación. Nota Técnica de Prevención. INSHT. http://www.mtas.es/insht/ntp/ntp_412.htm.

5. Priorities for occupational safety and health research in Europe: 2013-2020. European Agency for Safety and Health at Work, 2013;(1):13-20. http://europa.eu.

6. Verdes Y. El teletrabajo es casi inexistente en España. Aula TIC PYMEs de la USC. 2013. http://www.usc. es/atpemes/spip.php?auteur48.

7. Verano D, Suárez H, Sosa S. El teletrabajo y la mejora de la movilidad en las ciudades. Investigaciones Europeas de Dirección y Economía de la Empresa. 2013; http://dx.doi.org/10.1016/j.iedee.2013.03.002.

8. Rubio R. La transformación de los mercados laborales: el teletrabajo y sus alcances para el caso de Santiago, Chile. Revista de Geografía Norte Grande. 2010; (45):119-134.

9. Bustos OD. Sobre subjetividad y teletrabajo. Una revisión crítica. Revista de estudios sociales. 2012; (44):181-196.

10. Golden TD, Veiga JF, Dino RN. The impact of professional isolation on teleworker job performance and turnover intentions: does time spent teleworking, interacting face-to-face, or having access to communication-enhancing technology matter? J Appl Psychol. 2008 Nov; 93(6):1412-21.

11. Gajendran RS, Harrison DA. The good, the bad, and the unknown about telecommuting: meta-analysis of psychological mediators and individual consequences. J Appl Psychol. 2007 Nov; 92(6):1524-41.

12. Lapierre LM, Allen TD. Work-supportive family, family-supportive supervision, use of organizational benefits, and problem-focused coping: implications for work-family conflict and employee well-being. J Occup Health Psychol. 2006 Abril; 11(2):169-81.

13. Cifre E, Salanova M. El poder de la autoeficacia en la mejora de la salud psicosocial de la persona teletrabajadora. Salud Psicosocial. España. 2012: 71-98. cifre@uji.es; marisa.salanova@psi.uji.es.

14. Madero SM, Flores R. Predictores de la disposición de trabajadores mexicanos a aceptar el teletrabajo. Investigación y Ciencia. 2009; (43):46-52.

15. Sharit J, Czaja SJ, Hernandez MA et al. The Employability of Older Workers as Teleworkers: An Appraisal of Issues and an Empirical Study. Hum Factors Ergon Manuf. 2009; 19(5):457-477.

16. Fernandes A, De Dea K, Del Corso J. Telecommuting and HRM: a case study of an information technology service provider. JISTEM J.Inf.Syst.Technol.Manag. 2012; 2(9):285-306.

17. Gareca M, Verdugo R, Briones JL et al. Salud ocupacional y teletrabajo. Ciencia y trabajo. 2007 Sept; 9(25):85-88.

18. Harrington SS. Occupational Safety and Health. Training for Teleworkers: Findings from a Research Study Conducted for the National. Institute for Occupational Safety and Health. The Telework learning center. 2007:1-4. www.teleworktrainer.com.

19. Caamaño RE. El teletrabajo como una alternativa para promover y facilitar la conciliación de responsabilidades laborales y familiares. Revista de Derecho de la Pontificia Universidad Católica de Valparaíso. 2010; (7):79-105.

20. Robertson M, Schleifer L, Huang Y. Examining the macroergonomics and safety factors among teleworkers: Development of a conceptual model. Work. 2012; 41(1):2611-2615.

21. Fan C. Teleworker's home office: an extension of corporate office?. Facilities. 2010; 28(3):137-155.

22. Baker PMA, Moon NW, Ward AC. Virtual exclusion and telework: barriers and opportunities of technocentric workplace accommodation policy. Work. 2006; 27(4):421-30.

23. Kaplan S, Weiss S, Moon NW et al. A framework for providing telecommuting as a reasonable accommodation: some considerations on a comparative case study. Work. 2006; 27(4):431-40.

24. Nishina M. Applications of teleworking based on a study of disabled workers. Ind Health. 2010; 48(3):292-5.

25. Tausig M, Fenwick R. Unbinding time: Alternate work schedules and work-life balance. Journal of Family and Economic Issues. 2001; 22(2):101-119.

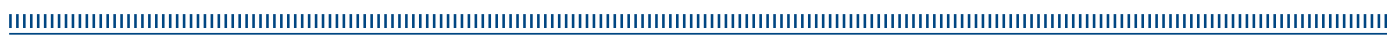

